\title{
Impact of NUDT15 genetics on severe thiopurine-related hematotoxicity in patients with European ancestry
}

\author{
Elke Schaeffeler, $\mathrm{PhD}^{1,2}$, Simon U. Jaeger, $\mathrm{MD}^{1,2}$, Verena Klumpp, MSc ${ }^{1,2}$, Jun J. Yang, PhD ${ }^{3,4,5}$, \\ Svitlana Igel, MD ${ }^{1,2}$, Laura Hinze ${ }^{6}$, Martin Stanulla, MD ${ }^{6}$ and Matthias Schwab, MD ${ }^{1,7,8}$
}

\begin{abstract}
Purpose: Severe hematotoxicity in patients with thiopurine therapy has been associated with genetic polymorphisms in the thiopurine S-methyltransferase (TPMT). While TPMT genetic testing is clinically implemented for dose individualization, alterations in the nudix hydrolase 15 (NUDT15) emerged as independent determinant of thiopurine-related hematotoxicity. Because data for European patients are limited, we investigated the relevance of NUDT15 in Europeans.
\end{abstract}

Methods: Additionally to TPMT phenotyping/genotyping, we performed in-depth Sanger sequencing analyses of NUDT15 coding region in 107 European patients who developed severe thiopurinerelated hematotoxicity as extreme phenotype. Moreover, genotyping for NUDT15 variants in 689 acute lymphoblastic leukemia (ALL) patients was performed.

Results: As expected TPMT was the main cause of severe hematotoxicity in $31 \%$ of patients, who were either TPMT deficient
(10\%) or heterozygous carriers of TPMT variants (21\%). By comparison, NUDT15 genetic polymorphism was identified in 14 (13\%) patients including one novel variant (p.Met1Ile). Six percent of patients with severe toxicity carried variants in both TPMT and NUDT15. Among patients who developed toxicity within 3 months of treatment, $13 \%$ were found to be carriers of NUDT15 variants.

Conclusion: Taken together, NUDT15 and TPMT genetics explain $\sim 50 \%$ of severe thiopurine-related hematotoxicity, providing a compelling rationale for additional preemptive testing of NUDT15 genetics not only in Asians, but also in Europeans.

Genetics in Medicine (2019) 21:2145-2150; https://doi.org/10.1038/s41436019-0448-7

Keywords: NUDT15; TPMT; pharmacogenetics; adverse drug reaction; hematotoxicity

\section{INTRODUCTION}

Thiopurine-related hematoxicity is clinically relevant and therapy-limiting in treatment of patients with acute lymphoblastic leukemia (ALL) or inflammatory bowel disease (IBD). In the past, retrospective and prospective clinical studies corroborated the clinical utility of genetic testing for thiopurine S-methyltransferase (TPMT) to avoid severe thiopurine-cytotoxicity. ${ }^{1-4}$ For clinical implementation, the Clinical Pharmacogenetics Implementation Consortium (CPIC) published peer-reviewed and evidence-based gene/ drug clinical practice guidelines ${ }^{5}$ indicating preemptive TPMT genotype-guided thiopurine-dose individualization to mitigate drug toxicity. Recently loss-of-function germline variants in NUDT15 have been identified as additional genetic determinants of thiopurine intolerance. ${ }^{6,7}$ Laboratory studies demonstrated excessive DNA damage and subsequent bone marrow suppression by the active thiopurine metabolite thioguanosine triphosphate (TGTP) in patients with NUDT15 deficiency. Inactivation of TGTP by converting TGTP to TGMP (thioguanosine monophosphate) is regulated by the nucleotide diphosphatase activity of NUDT15, thereby counteracting the cytotoxic effects of thiopurines. Consistently, thiopurine-treated Nudt15 knock-out mice also develop severe leukopenia compared with wild-type mice. ${ }^{8}$

So far the impact of NUDT15 on thiopurine hematotoxicity as an extreme phenotype in populations other than Asians is limited because NUDT15 variants are most prevalent in Asians. ${ }^{6,9}$ However, first reports indicate that for instance TPMT and NUDT15 genes are both related to thiopurinerelated toxicity in patients from Uruguay. ${ }^{10}$ In the present paper, we evaluate the importance of NUDT15 genetics, in addition to TPMT variation, for development of severe hematotoxicity in thiopurine-treated patients with European ancestry.

\footnotetext{
${ }^{1}$ Dr. Margarete Fischer-Bosch-Institute of Clinical Pharmacology, Stuttgart, Germany; ${ }^{2}$ University of Tübingen, Tübingen, Germany; ${ }^{3}$ Department of Pharmaceutical Sciences, St. Jude Children's Research Hospital, Memphis, TN, USA; ${ }^{4}$ Department of Oncology, St. Jude Children's Research Hospital, Memphis, TN, USA; ${ }^{5} \mathrm{Hematological} \mathrm{Malignancies}$ Program, Comprehensive Cancer Center, St. Jude Children's Research, Hospital, Memphis, TN, USA; ${ }^{6}$ Pediatric Hematology and Oncology, Hannover Medical School, Hannover, Germany; ${ }^{7}$ Department of Clinical Pharmacology and Pharmacy and Biochemistry, University Tübingen, Tübingen, Germany; ${ }^{8}$ Departments of Clinical Pharmacology and Biochemistry, Yerevan State Medical University, Yerevan, Armenia. Correspondence: Matthias Schwab (matthias.schwab@ikp-stuttgart.de)
} 
a

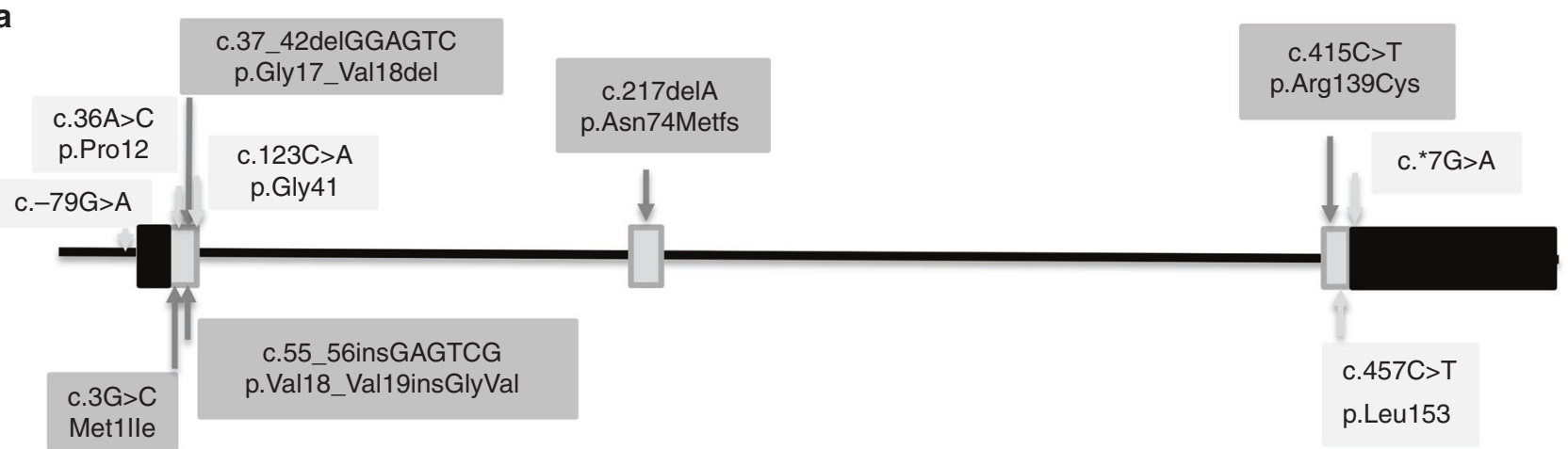

b

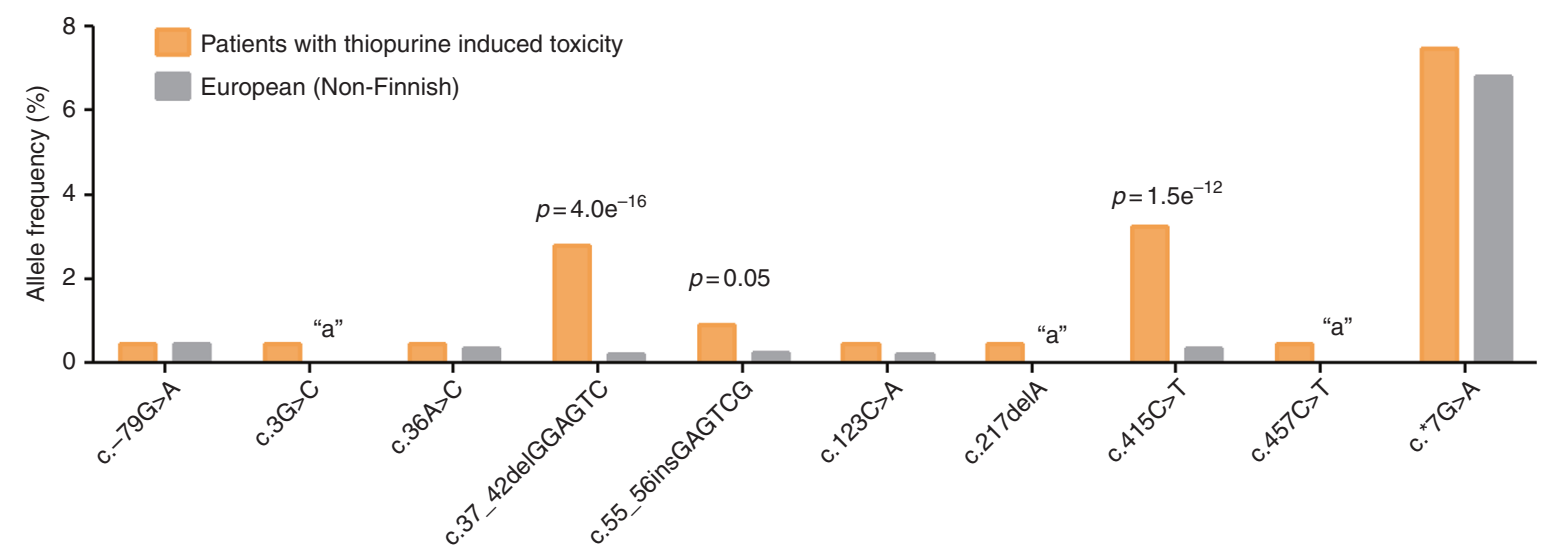

C All cases $(n=107)$

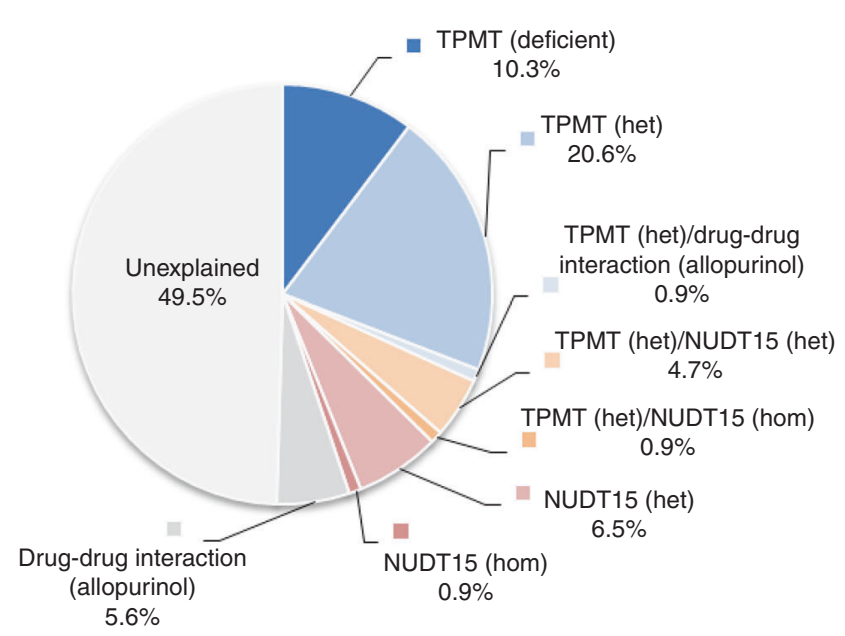

Cases $(n=47)$ who developed toxicity $\leq 3$ months

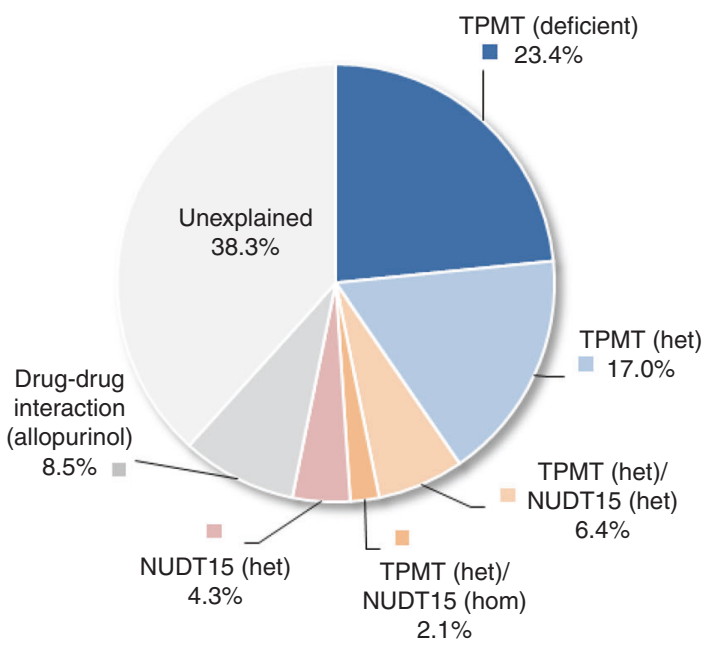

Fig. 1 NUDT15 genetics and thiopurine-related hematotoxicity (a) Genetic variants detected in the NUDT15 gene region in patients with severe thiopurine-related toxicity. Dark gray arrows indicate variants resulting in variation of the NUDT15 protein sequence. Boxes indicate exons 1-3, and untranslated regions are shown in black. (b) Allele frequency of NUDT15 variants in patients with thiopurine-induced toxicity and individuals of European (non-Finnish) ancestry extracted from the Genome Aggregation Database (gnomAD) ${ }^{14}$ (see Table S3). $P$ values indicate significant differences in genotype frequency as tested by the Armitage's trend test. ${ }^{a}$ Variants are not reported in exome or genome sequencing data of gnomAD. ${ }^{14}$ (c) Pie chart illustrates percentage of patients with variation in TPMT or NUDT15 genetics as well as percentage of patients with allopurinol treatment. Left part shows fractions in all patients $(n=107)$, whereas fractions in patients $(n=47)$ who developed toxicity within 3 months after the first administration of therapy are illustrated on the right.

MATERIALS AND METHODS

The study cohort $(n=180)$ comprised of consecutively included patients who experienced severe clinical thiopurine-related cytopenia/pancytopenia and were tested for TPMT at the pharmacogenetic laboratory at our institute between January 2002 and September 2006. Thiopurine hematotoxicity was 
documented by the responsible physician in a standardized questionnaire and/or if available on hematological laboratory criteria as previously published ${ }^{11}$ and in line with accepted criteria (https://ctep.cancer.gov/protocolDevelopment/ electronic_applications/docs/ctcaev3.pdf). Only patients with a sufficient amount of remaining DNA $(n=107)$ and patient characteristics were selected for analyses of NUDT15 genetics through Sanger sequencing (see supplementary materials and methods). Further information about patient characteristics and thiopurine treatment is given in Table S1. The study was approved by the local ethics committee of the University of Tübingen, Germany, and all analyses were done anonymously.

The second cohort included ALL children $(n=689)$ treated according to the Berlin-Frankfurt-Münster (BFM) trial AIEOP-BFM ALL 2009 (ref. ${ }^{12}$ ) and was used as an independent control group of 6-mercaptopurine (6-MP) treated patients with preemptive genotyping for TPMT and subsequent 6-MP dose adjustment only in case of TPMT deficiency to avoid thiopurine-related hematotoxicity. ${ }^{11,13}$ For details, see Table S2. The study was approved by the ethical review board and written informed consent was provided. Further details about the method used for NUDT15 and TPMT genotyping are provided in supplementary materials and methods.

Detailed information about statistical methods used to compare frequency distributions with the general European population extracted from the Genome Aggregation Database $(\text { gnomAD })^{14}$ is given in supplementary materials and methods.

\section{RESULTS}

Although previous studies suggested that NUDT15 genetics is associated with thiopurine-related hematotoxicity especially in Asians, only limited data are available for patients of European ancestry. Therefore, we performed the first analysis of genetic variability of NUDT15 in 107 European patients who developed severe thiopurine-associated hematotoxicity. The study cohort consisted mainly of patients with inflammatory bowel diseases $(n=68)$ or other autoimmune diseases. The median dosage of azathioprine and 6-MP was $100 \mathrm{mg}$ (range 50 to $300 \mathrm{mg}$ ) and $50 \mathrm{mg}$ (50 to $125 \mathrm{mg}$ ), respectively (Table S1). In total, ten different genetic variants were found within NUDT15 by Sanger sequencing covering all exonic and flanking intronic regions (Fig. 1a). All ten genetic variants and their frequency distribution in 107 individuals are listed in Table S3. In the exonic regions, eight variants were found, of which five were missense variants (see Table S3). Three of these missense variants have previously been associated with thiopurine-related toxicity (p.Gly17_Val18del, p.Val18_Val19insGlyVal, p.Arg139Cys). ${ }^{6,7}$ To further investigate an association of these variants with toxicity, we compared their frequencies in our study cohort with the frequency present in the general European population. Here, two of these variants were strongly (p.Gly17_Val18del: $p=4.0 \mathrm{e}^{-16}$ and p.Arg139Cys: $p=1.5 \mathrm{e}^{-12}$ ) and one moderately (p.Val18_Val19insGlyVal: $p=0.05$ ) significantly more frequent in our patient cohort compared with the general European population (Fig. 1b), which further supports an association of these variants with thiopurine toxicity and functional consequences. In addition, we identified one novel variant resulting in the loss of the start codon (c.3G>C, NUDT15 $\left.{ }^{*} 19\right)$ and one missense frameshift variant (c.217delA, NUDT15 18 ), both with consequences on NUDT15 function and activity due to severe alterations in the NUDT15 protein sequence. Moreover, both of these variants seem to be rare and were not detected in exome or genome sequencing data in the European (non-Finnish) population (gnomAD, ${ }^{14}$ see Table S3). Genotyping of the most frequent functional relevant variants, p.Gly17_Val18del and p. Arg139Cys, in a cohort of ALL patients revealed that both the p.Gly17_Val18del and p.Arg139Cys variant were absent in this cohort of ALL patients, indicating their very low frequency in patients of European ancestry (Table S3). Because the frequencies of the genetic variants detected in the 5'UTR or 3'UTR were not significantly different between patients who developed toxicity and the European population, it is unlikely that they are linked to functional consequences resulting in thiopurine toxicity (Fig. 1b). Moreover, synonymous variants resulting in no alterations of NUDT15 protein sequence most likely do not impair NUDT15 function. Thus only the five missense variants described above were considered as candidate variants for the explanation of thiopurine-associated toxicity in our patient cohort. In total, $14(13 \%)$ of the 107 patients carry at least one of these variants. The characteristics of these patients together with their NUDT15 genotypes/haplotypes as well as detailed information about thiopurine treatment are summarized in Table 1. Because it is well established that TPMT pheno- or genotype is associated with thiopurine-related hematotoxicity, all patients of our study were phenotyped or genotyped for the most frequent inactivating TPMT alleles (see supplementary materials and methods). Of note, 6 of 14 patients who carry at least one NUDT15 missense variant are also carriers of one TPMT allele responsible for reduced TPMT activity (Table 1). As shown in Fig. 1c (left panel), among all 107 patients, $11(10 \%)$ were TPMT deficient and $22(21 \%)$ carried one TPMT variant allele. To evaluate the impact of comedication on the development of toxicity, we solely investigated cotreatment with allopurinol. Allopurinol is the only drug for which dose adjustment to avoid hematotoxicity is recommended by the prescription information of thiopurines. ${ }^{15,16}$ In our cohort, seven patients received allopurinol as comedication without adequate dose adjustment of azathioprine. One of these patients was also $T P M T^{*} 1 /{ }^{*} 3 A$. In $50 \%$ of patients the occurrence of toxicity could not be explained by both TPMT and NUDT15 genetics, or by allopurinol medication. Considering only patients $(n=47)$ who developed hematotoxicity within 3 months after onset of thiopurines indicated that $13 \%$ of these patients carry at least one NUDT 15 variant and $53 \%$ of these patients are carriers of at least one NUDT15 or TPMT variant (Fig. 1c, right panel). 


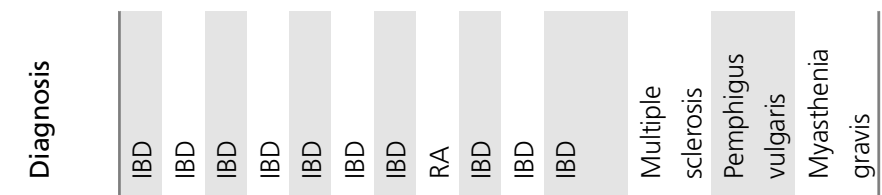

离

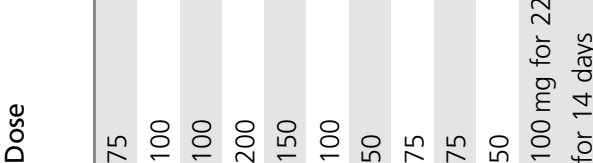

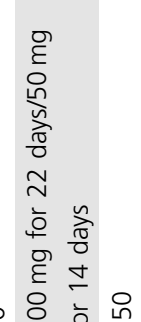

$\frac{\widehat{a}}{\frac{2}{0}}$

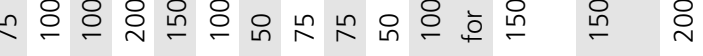

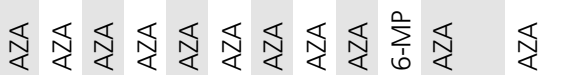
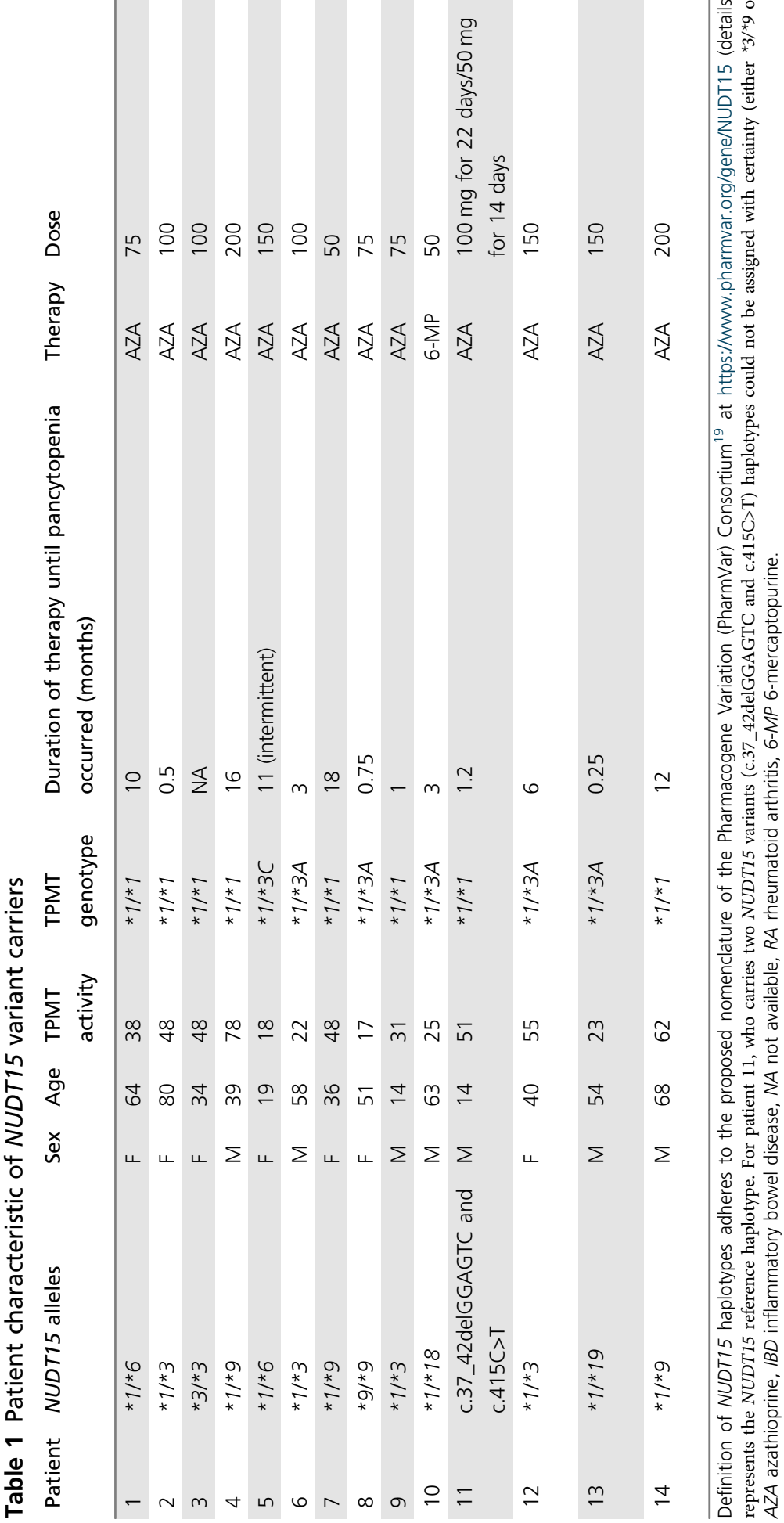
Of note, all 11 TPMT deficient patients in our cohort developed toxicity within 3 months. Taken together, toxicity in $38 \%$ of patients remained unexplained.

\section{DISCUSSION}

Thiopurine-associated hematotoxicity has so far been mainly attributed to alterations in TPMT, but the role of NUDT15 especially in Europeans remains elusive. Therefore, we performed for the first time a comprehensive analysis of genetic variability of NUDT15 in European patients $(n=107)$ with different diseases, who developed severe thiopurinerelated hematotoxicity documented by a standardized questionnaire and/or hematological laboratory. ${ }^{11}$ In summary, we identified through comprehensive sequencing ten different genetic variants within NUDT15, including five missense variants (see Table S3). Three of these missense variants have already been associated with thiopurine-related toxicity ( $p$. Gly17_Val18del, p.Val18_Val19insGlyVal, p.Arg139Cys), which was corroborated by studies investigating their functional consequences. ${ }^{6,7}$ These findings are further supported by our study, indicating a significantly higher frequency of these variants in our patient cohort representing an extreme phenotype (severe thiopurine-related hematotoxicity) compared with the general European population (Fig. 1b). In addition, two rare missense variants in NUDT15 were detected, which have not been associated with thiopurine-related hematotoxicity so far. Of note, both variants have functional consequences resulting either in the loss of the start codon (c.3G>C,NUDT15 $\left.{ }^{\star} 19\right)$ or in a frameshift (c.217delA, NUDT15*18). Thus our findings provide first evidence that rare functional variants within NUDT15 exist in Europeans, which supports the relevance for in-depth sequencing in patients with European ancestry prior to thiopurine therapy to avoid thiopurine-related hematotoxicity.

Because severe hematotoxicity in patients with thiopurine therapy (e.g., azathioprine, 6-mercaptopurine) is associated with genetic polymorphisms in TPMT, all patients in our study were not only phenotyped, but also genotyped for relevant TPMT alleles as well. Thereby we confirmed that TPMT was the main cause of severe hematotoxicity in $31 \%$ of patients, who were either TPMT deficient $(10 \%)$ or heterozygous carriers of TPMT variants (21\%). Regarding NUDT15, functionally relevant missense variants were identified in 14 (13\%) patients, of whom 6 patients with severe toxicity carried variants in both TPMT and NUDT15 (Fig. 1c, left panel). Thus the combination of impaired NUDT15 and TPMT activity might further increase the probability for developing thiopurine-related toxicity compared with deficiency in only one of the two genes. Generally, toxicity occurred at median within 4 months, but a broad range up to 120 months was observed. This long period was observed in one patient with IBD and TPMT heterozygosity receiving $60 \%$ of the recommended standard dose. In spite of dose reduction, over the long term $60 \%$ of dosage appears to be still too high, corroborating individual thiopurine dosing
(40\% up to $70 \%$ of standard dose) in TPMT heterozygous patients. Because severe toxicity due to genetic reasons most likely occurs within a few months, ${ }^{2}$ we performed a subanalysis for the association of TPMT and NUDT15 genetics and thiopurine-related toxicity including only those patients who developed toxicity within 3 months. In total, among patients who developed toxicity within 3 months of treatment, $53 \%$ could be explained by TPMT and/or NUDT15 genetics, but $38 \%$ remained unexplained, also considering comedication (Fig. 1c, right panel). Because of their normal TPMT activity (median: 42.0 [24-72]) (refs. ${ }^{13,17}$ ), rare genetic variants within TPMT that were not tested in the present study can be excluded to explain toxicity. Because thiopurinerelated hematotoxicity is multifactorial, additional risk factors (e.g., viral infection) or novel risk genes might be responsible for toxicity in these patients. Our study has some limitations. First, it is a retrospective analysis. Second, a potential bias of the study results may be the selection of only 107 of 180 patients reported with severe thiopurine-related hematotoxicity. However, because all patients for pharmacogenetic diagnostics at our center were consecutively collected and the frequency distribution of heterozygous and homozygous TPMT variant carriers is comparable in both groups of patients $(p=0.32$; see Table S4), we believe the bias is negligible.

In summary, we provide evidence that NUDT15 genetics in addition to TPMT is a critical factor contributing to thiopurine-induced hematotoxicity in patients with European ancestry. Although prospective studies and health economic evaluations are currently missing, the process to implement NUDT15 genetics in clinical practice will most likely be facilitated by the upcoming guideline of the CPIC. ${ }^{18}$ Our data indicate that NUDT15 variants are worth implementing in preemptive pharmacogenetic testing in clinical practice for genotype-guided thiopurine therapy.

\section{SUPPLEMENTARY INFORMATION}

The online version of this article (https://doi.org/10.1038/s41436019-0448-7) contains supplementary material, which is available to authorized users.

\section{ACKNOWLEDGEMENTS}

We gratefully acknowledge Monika Elbl and Andrea Jarmuth for excellent technical assistance. This study was supported in part by the Robert Bosch Stiftung Stuttgart, the Horizon 2020-PHC-2015 grant U-PGX 668353, and the ICEPHA Graduate School Tübingen-Stuttgart. The authors would like to thank the Genome Aggregation Database (gnomAD) and the groups that provided exome and genome variant data to this resource. A full list of contributing groups can be found at http://gnomad. broadinstitute.org/about.

\section{DISCLOSURE}

The authors declare no conflicts of interest. 
Publisher's note: Springer Nature remains neutral with regard to jurisdictional claims in published maps and institutional affiliations.

\section{REFERENCES}

1. Coenen MJ, Jong DJ, de, van Marrewijk CJ, et al. Identification of patients with variants in TPMT and dose reduction reduces hematologic events during thiopurine treatment of inflammatory bowel disease. Gastroenterology. 2015;149:907-917.

2. Colombel JF, Ferrari N, Debuysere H, et al. Genotypic analysis of thiopurine S-methyltransferase in patients with Crohn's disease and severe myelosuppression during azathioprine therapy. Gastroenterology. 2000;118:1025-1030.

3. Schwab M, Schaeffeler E, Marx C, et al. Azathioprine therapy and adverse drug reactions in patients with inflammatory bowel disease: impact of thiopurine S-methyltransferase polymorphism. Pharmacogenetics. 2002;12:429-436.

4. Relling MV, Hancock ML, Rivera GK, et al. Mercaptopurine therapy intolerance and heterozygosity at the thiopurine S-methyltransferase gene locus. J Natl Cancer Inst. 1999;91:2001-2008.

5. Relling MV, Gardner EE, Sandborn WJ, et al. Clinical pharmacogenetics implementation consortium guidelines for thiopurine methyltransferase genotype and thiopurine dosing: 2013 update. Clin Pharmacol Ther. 2013;93:324-325.

6. Moriyama T, Nishii R, Perez-Andreu V, et al. NUDT15 polymorphisms alter thiopurine metabolism and hematopoietic toxicity. Nat Genet. 2016;48:367-373.

7. Moriyama T, Yang Y-L, Nishii R, et al. Novel variants in NUDT15 and thiopurine intolerance in children with acute lymphoblastic leukemia from diverse ancestry. Blood. 2017;130:1209-1212.

8. Nishii R, Moriyama T, Janke $\sqcup$, et al. Preclinical evaluation of NUDT15guided thiopurine therapy and its effects on toxicity and antileukemic efficacy. Blood. 2018;131:2466-2474.

9. Cargnin S, Genazzani AA, Canonico PL, Terrazzino S. Diagnostic accuracy of NUDT15 gene variants for thiopurine-induced leukopenia: a systematic review and meta-analysis. Pharmacol Res. 2018;135:102-111.

10. Soler AM, Olano N, Méndez Y, et al. TPMT and NUDT15 genes are both related to mercaptopurine intolerance in acute lymphoblastic leukaemia patients from Uruguay. Br J Haematol. 2018;181:252-255.

11. Stanulla M, Schaeffeler E, Flohr T, et al. Thiopurine methyltransferase (TPMT) genotype and early treatment response to mercaptopurine in childhood acute lymphoblastic leukemia. JAMA. 2005; 293:1485-1489.
12. Lanvers-Kaminsky $C$, Rüffer $A$, Würthwein $G$, et al. Therapeutic drug monitoring of asparaginase activity-method comparison of MAAT and AHA test used in the International AIEOP-BFM ALL 2009 Trial. Ther Drug Monit. 2018;40:93-102.

13. Tamm R, Mägi $R$, Tremmel $R$, et al. Polymorphic variation in TPMT is the principal determinant of TPMT phenotype: a meta-analysis of three genome-wide association studies. Clin Pharmacol Ther. 2017;101:684-695.

14. Lek M, Karczewski KJ, Minikel EV, et al. Analysis of protein-coding genetic variation in 60,706 humans. Nature. 2016;536:285-291.

15. https://www.accessdata.fda.gov/drugsatfda_docs/label/2011/ 009053s032lbl.pdf. Accessed 4 February 2019.

16. https://www.accessdata.fda.gov/drugsatfda_docs/label/2011/ 016324s034s035lbl.pdf. Accessed 4 February 2019.

17. Schaeffeler E, Fischer C, Brockmeier D, et al. Comprehensive analysis of thiopurine S-methyltransferase phenotype-genotype correlation in a large population of German-Caucasians and identification of novel TPMT variants. Pharmacogenetics. 2004;14:407-417.

18. Relling MV, Schwab M, Whirl-Carrillo M, et al. Clinical Pharmacogenetics Implementation Consortium (CPIC) guideline for thiopurine dosing based on TPMT and NUDT15 genotypes: 2018 update. Clin Pharmacol Ther. 2018. https://doi.org/10.1002/cpt.1304. Accessed 4 February 2019.

19. Yang J, Whirl-Carrillo M, Scott SA, et al. Pharmacogene Variation (PharmVar) Consortium gene introduction: NUDT15. Clin Pharmacol Ther. 2018. https://doi.org/10.1002/cpt.1268. Accessed 4 February 2019.

(i) $\risingdotseq$ Open Access This article is licensed under a Creative Commons c. Attribution-NonCommercial-NoDerivatives 4.0 International License, which permits any non-commercial use, sharing, distribution and reproduction in any medium or format, as long as you give appropriate credit to the original author(s) and the source, and provide a link to the Creative Commons license. You do not have permission under this license to share adapted material derived from this article or parts of it. The images or other third party material in this article are included in the article's Creative Commons license, unless indicated otherwise in a credit line to the material. If material is not included in the article's Creative Commons license and your intended use is not permitted by statutory regulation or exceeds the permitted use, you will need to obtain permission directly from the copyright holder. To view a copy of this license, visit http://creativecommons.org/licenses/by-nc-nd/4.0/.

(C) The Author(s) 2019 